\title{
Privacy and Usability of Image and Text Based Challenge Questions Authentication in Online Examination
}

\author{
Abrar Ullah, Hannan Xiao, Mariana Lilley, Trevor Barker \\ School of Computer Science, \\ University of Hertfordshire, Hatfield, UK \\ a.ullah3, h.xiao,m.lilley, t.1.barker@herts.ac.uk
}

\begin{abstract}
In many online examinations, physical invigilation is often replaced with traditional authentication approaches for student identification. Secure and usable authentication approaches are important for high stake online examinations. A Profile Based Authentication Framework (PBAF) was developed and implemented in a real online learning course embedded with summative online examination. Based on users' experience of using the PBAF in an online course, online questionnaires were used to collect participants' feedback on effectiveness, layout and appearance, user satisfaction, distraction and privacy concerns. Based on overall findings of the quantitative analysis, there was a positive feedback on the use of a hybrid approach utilizing image and text based challenge questions for better usability. However, the number of questions presented during learning and examination processes were reported to be too many and caused distraction. Participants expressed a degree of concern on sharing personal and academic information with little or no privacy concern on using favorite questions.
\end{abstract}

Keywords-Online examination, profile, challenge questions, authentication, security, usability, traffic light system

\section{INTRODUCTION}

Online examination is an important and critical part of online learning. Secure examination is essential for credibility of online learning due to inherent absence of physical invigilation in many online examinations. Student identification is largely reliant upon remote authentication approaches and therefore secure authentication is essential for secure examination.

The authors developed and presented the PBAF method for authentication of students in online examination [17]. The PBAF is a knowledge-based authentication approach to combine traditional passwords and challenge questions. This method utilizes predefined challenge questions to integrate learning and examination processes.

In earlier studies, we implemented the PBAF method in a simulation online learning environment for evaluation of usability and security [16], [19], [18].This study is part of ongoing research to evaluate usability and privacy attributes from users feedback after using the PBAF method in a real online course. The study was performed in two main phases. In the first phase a free "PHP and MySQL" course was setup with summative online examination (weekly quizzes) using the PBAF method for student authentication. In the second phase, an online survey questionnaire was used to collect participants feedback on their experience of using the PBAF method. The findings presented in this paper are based on the second phase of the empirical study. The online questionnaire was based on important usability factors including effectiveness, layout and appearance, user satisfaction and distraction [8]. Earlier studies on challenge questions raised users 'concerns on the use of personal information for authentication purposes. Therefore, this study collected participants' feedback using online survey on their privacy concerns for all challenge questions used in a "PHP and MySQL "online course using the PBAF methods.

\section{BACKGROUND}

In many online learning environments, online examination is an important feature and a critical asset [6]. A number of previous studies acknowledged that student authentication in online examination faces many security threats. Unethical conduct has been growing in online learning due to uncontrolled environment in online examinations as a result of use of technology and the Internet [1],[3]. In spite of a number of benefits, online learning is reported to be vulnerable to many security threats due to high stake summative examinations. In online environments, student identification is largely reliant upon authentication approaches due to inherent anonymity of users on the web. A number of authentication features have evolved over time to deliver usable, secure and reliable examination process. These authentication features have their own benefits and limitations in terms of cost, wider compatibility and security.

The PBAF is a multi-modal knowledge based approach, which collects answers to pre-defined questions and build a user's profile during the learning process recurrently. The profile information is used for user's authentication during examination process. A better question design is essential for secure implementation of the PBAF approach.

Challenge questions authentication is widely used for credential recovery and secure transactions in the online banking sectors [12],[10]. Traditionally, challenge questions uses fixed or open type text based questions for authentication purposes [18]. In an earlier empirical study [16], we implemented fixed type text based challenge questions. However, due to usability challenges reported in a number of studies [4],[5], [17],[19], we have used recall and recognition 
based image questions in the PBAF. Stemming from peoples ability to remember pictures over words, the PBAF implemented various graphical password strategies. This study reports on the usability of text and image hybrid questions after analyzing the results from an online questionnaire. The idea of trying to replace challenge questions is an ongoing problem. The method of authenticating with images over words can be a usable and memorable approach when compared to traditional text-based authentication. On their own, graphical passwords do have weaknesses, such as shoulder surfing, therefore, text based questions were combined with graphical approaches which may alleviate this concern.

Usability is an important factor for better authentication features. Students are particularly focused on their learning in online environments. Any additional and less usable interruptions for authentication may fraustate students. Therefore, it is important to evaluate their feedback on usability of the PBAF method.

Users of web applications are particularly concerned about their information privacy [15]. In the context of PBAF using challenge questions, this may increase their concern, if questions invade personal and private space. It is important to understand users' willingness to share their personal information, which can be perceived as private. Therefore, participants' feedback on usability and privacy was collected and presented in this paper

\section{PROFILE BASED AUTHENTICATION CONTEXT}

The PBAF is a multi-modal authentication approach implemented on MOODLE Learning Management System (LMS).

Students are provided with a unique username and password for logging into the learning environment. Upon successful login, students are asked to answer profile questions in order to gain access to learning resources. The profile questions are used to collect answers in order to build and update their individual profiles. The authentication process is triggered when a student requests to access an online examination. The student is then redirected to answer a set of challenge questions randomly selected from their profile.

\section{RESEARCH METHODOLOGY}

The study was organized into two phases with the second phase reported here. The second phase of user study was based on first phase which was organized into multiple sub phases including participants' recruitment, course setup, challenge questions design, course induction. The second phase involved quantitative analysis using questionnaire design and instrumentation, participants' recruitment from the first phase and conducting an online survey. The questionnaire was setup in an online survey tool. The study design and methodology was approved by the University of Hertfordshire research ethics committee.

\section{A. Online Questionnaire Instrumentation}

The In this research, 19- instruments online questionnaire was designed to collect students' feedback on various usability attributes of the PBAF method. One of the primary objectives of the questionnaire was collection of feedback based on Nielsen Heuristics [7] and International Organization of Standard (ISO) usability metrics [14] to evaluate ease of use, satisfaction, distraction from the learning process, layout and appearance, readability of questions, visual interface and size of images used in image questions, comparison of image and text based. For usability evaluation, 5 points Likert scale [1-5] [Strongly Disagree -Strongly Agree] was framed. Statement 11 was evaluated on scale [1-5] indicating [Too Few, Few, Just Right, Many, Too Many].

For privacy evaluation 28-instruments online questionnaire was designed for challenge questions used in the first phase of PBAF implementation. For privacy feedback, three point "concern" scale [1-3] indicating [1=A greet privacy concern, $2=$ Slight privacy concern, $3=$ No privacy concern] was implemented.

\section{B. Participants Recruitement}

A blend of local and international undergraduate and postgraduate students were recruited from UK and overseas Universities. In the first phase of user study, a total of 70 students were recruited in order to support research and learning activities simultaneously. The distribution of sample size was not uniform across the countries and cities, but there was a good representation from a diverse group of students from 9 countries with a large number of 50(71\%) students from United Kingdom. A total of 11(16\%) students from Pakistan, 2(4\%) students from Malta and Nigeria, $1(1 \%)$ each from Ireland, Greece, India, Trinidad and Tobago, and Togo attended the online course. The second phase of user study was attended by 31 of the total 70 participants.

In phase one of user study, a free "PHP and MySQL" online course was designed and deployed on MOODLE LMS. The PBAF method was built and integrated in the LMS for authentication of participants in 5 weekly quizzes. A total of 28 text-based and 13 image based questions was used for implementation of the PBAF. On completion of online course, participants were requested to submit their feedback using an online survey. Feedback from the survey is analysed and discussed in the section below.

\section{RESUlTS}

It was 19 -instrument survey to evaluate, effectiveness, degree of user satisfaction based on heuristic and usability metrics, distraction from actual learning and privacy after using PBAF method in an online course. The central tendency and dispersion of participants' feedback was analysed. Mean and median score were calculated to measure the central tendency and standard deviation was computed to measure dispersion.

\section{A. Usability Results}


The usability results presented in Table 1 are extracted from participants' feedback to an online questionnaire. Column 1 shows survey questions and columns 2-6 shows the percentage of participants' feedback on a scale of 1-5 defined earlier. Columns 7-9 shows central tendency and dispersion under Mean (M), Median (MD) and Standard Deviation (SD).

\section{1) Effectiveness}

Effectiveness is a degree of accuracy and an important usability attribute. In the context of this study, participants were requested for their feedback on the effectiveness of the PBAF and challenge questions in statements 1-3. The first statement requested participants' feedback on the degree of ease while using the PBAF method. The mean score is positive with $(55+23) 78 \%$ agreement. The profile question collection was a recurrent step and it was imperative to enquire participants' feedback to measure the effectiveness of the PBAF. The second and third statements enquired participants' acceptance of additional steps while answering text and image based profile questions. The usability of text-based questions received $(45+26) 71 \%$ agreement from participants and scored 3.8, whereas statement regarding image-based questions received $(42+35) 77 \%$ agreement with $35 \%$ strong agreement. The results show user acceptance of the PBAF approach based on their experience of using it in an online course. However, some participants disagreed or remained neutral in their feedback which indicates improvement in areas not reported in this work.

Table 1 Usability Survey Results

\begin{tabular}{|c|c|c|c|c|c|c|c|c|c|}
\hline \multirow[t]{2}{*}{ No. } & \multirow[t]{2}{*}{ Survey Statement } & \multicolumn{5}{|c|}{$\begin{aligned} \% \text { of }[1 & =\text { Strongly Disagree to } \\
5 & =\text { Strong Agree }]\end{aligned}$} & \multicolumn{3}{|c|}{$\begin{array}{l}\text { Central Tendency and Dispersion } \\
(n=31)\end{array}$} \\
\hline & & 1 & 2 & 3 & 4 & 5 & M & MD & SD \\
\hline \multicolumn{10}{|c|}{ Effectiveness } \\
\hline 1 & PBAF method was easy to use & 0 & 16 & 6 & 55 & 23 & 3.8 & 4 & 1.0 \\
\hline 2 & $\begin{array}{l}\text { I could effectively carry on with the } \\
\text { learning activities while answering the text } \\
\text { based security questions }\end{array}$ & 0 & 16 & 13 & 45 & 26 & 3.8 & 4 & 1.0 \\
\hline 3 & $\begin{array}{l}\text { I could effectively carry on with the } \\
\text { learning activities while answering the } \\
\text { image questions }\end{array}$ & 0 & 13 & 10 & 42 & 35 & 4.0 & 4 & 1.0 \\
\hline \multicolumn{10}{|c|}{ Appearance and Layout } \\
\hline 4 & $\begin{array}{l}\text { The interface of this system was } \\
\text { aesthetically pleasant }\end{array}$ & 3 & 6 & 23 & 55 & 13 & 3.7 & 4 & 0.9 \\
\hline 5 & $\begin{array}{l}\text { I am satisfied with the layout of profile } \\
\text { questions }\end{array}$ & 0 & 6 & 23 & 61 & 10 & 3.7 & 4 & 0.7 \\
\hline 6 & $\begin{array}{l}\text { Overall, I am satisfied with the layout of } \\
\text { image and text based challenge questions }\end{array}$ & 0 & 0 & 10 & 61 & 29 & 4.2 & 4 & 0.6 \\
\hline 7 & The questions were readable & 0 & 0 & 0 & 61 & 39 & 4.4 & 4 & 0.5 \\
\hline 8 & The questions were easy to understand & 0 & 0 & 0 & 61 & 39 & 4.4 & 4 & 0.5 \\
\hline 9 & $\begin{array}{l}\text { The on-screen captions provided with this } \\
\text { system were clear }\end{array}$ & 0 & 3 & 7 & 74 & 16 & 4.0 & 4 & 0.6 \\
\hline \multicolumn{10}{|c|}{ Distraction } \\
\hline 10 & $\begin{array}{l}\text { I was distracted by the profile questions } \\
\text { asked after the login }\end{array}$ & 10 & 35 & 19 & 23 & 13 & 2.9 & 3 & 1.2 \\
\hline 11 & $\begin{array}{l}{ }^{*} \text { The number of questions asked after the } \\
\text { login was }\end{array}$ & 0 & 6 & 42 & 29 & 23 & 3.7 & 4 & 0.9 \\
\hline 12 & $\begin{array}{l}\text { I am satisfied with the number of security } \\
\text { questions asked before access to weekly } \\
\text { quizzes }\end{array}$ & 16 & 26 & 10 & 45 & 3 & 2.9 & 3 & 1.2 \\
\hline \multicolumn{10}{|c|}{ Satisfaction } \\
\hline 13 & $\begin{array}{l}\text { I was satisfied with the size of images in } \\
\text { image questions }\end{array}$ & 0 & 0 & 6 & 65 & 29 & 4.2 & 4 & 0.6 \\
\hline 14 & $\begin{array}{l}\text { I was satisfied with the quality of images in } \\
\text { image questions }\end{array}$ & 0 & 0 & 13 & 61 & 26 & 4.1 & 4 & 0.6 \\
\hline 15 & $\begin{array}{l}\text { Overall, I am satisfied with the appearance } \\
\text { of image and text based challenge questions }\end{array}$ & 0 & 3 & 16 & 58 & 23 & 4.0 & 4 & 0.7 \\
\hline 16 & $\begin{array}{l}\text { Overall, I am satisfied with the security } \\
\text { questions based system used in the online } \\
\text { course }\end{array}$ & 0 & 29 & 13 & 42 & 16 & 3.5 & 4 & 1.1 \\
\hline 17 & The image questions were clear & 0 & 0 & 13 & 58 & 29 & 4.2 & 4 & 0.6 \\
\hline \multicolumn{10}{|c|}{ Image Based Questions } \\
\hline 18 & I liked the idea of using image questions & 0 & 13 & 16 & 39 & 32 & 3.9 & 4 & 1.0 \\
\hline 19 & $\begin{array}{l}\text { I preferred image questions over text } \\
\text { questions }\end{array}$ & 3 & 13 & 32 & 16 & 36 & 3.7 & 4 & 1.2 \\
\hline
\end{tabular}




\section{2) Layout and Appearance.}

The application interface consisting of layout and appearance is a highly important aspect of the system design process. It interfaces end users and application processes. The layout and appearance is an essential factor to inform usability aspect of the PBAF. Nielsen's [7] guidelines for user interface design indicates the importance of standardization in layout, aesthetically pleasant interface and proper image sizes.

Students were requested to submit their feedback on aesthetic design, layout of profile questions and layout of text and image based questions in statements 4-6. The survey statements in 4-5 regarding aesthetic design and layout of profile questions received an overall positive score. However, some participants remained neutral or in disagreement with the statement, which indicates further improvement in the layout. Participants' feedback to statement in 6 scored 4.2 with a significant degree of $(61+29) \quad 90 \%$ agreement to overall satisfaction on layout and appearance of the PBAF processes.

Nielsen and Tahir [9] suggest using simple, non-technical and customer focused language for writing captions, labels and contents. Survey statements 7-9 recorded feedback on the language and readability of questions. The feedback was significantly positive with $(61+39) \quad 100 \%$ agreement on language readability and understanding due to better question design.

\section{3) Distraction}

The PBAF method introduced additional processes for recording profile questions during learning and posing challenge questions for authentication in online examination. Shneiderman and Ben's [13] indicate that additional processes can frustrate users. Participants' feedback on distraction during learning process was collected in statements10-12. Statement 10 requested "distracted by profile questions" scored 2.9 with $(23+13) 36 \%$ agreement. There was a $19 \%$ neutral score and the findings indicate a degree of distraction caused by posing profile questions in their learning process. Recording profile question is an important part of the PBAF approach, however, to minimize distraction this could be replaced with alternative such as learning activity based questions. Statement 11 requested feedback on the number of profile questions. The number of profile question collected after login was set to 3 and participants' feedback indicate this to be too many. A total of $48 \%$ participants think the number of questions to be "Just Right" or "Low", however, (29+23) 52\% expressed a concern on the number questions to be "Many" or "Too Many". Statement 12 also received (12+26) 42\% disagreement on the number of challenge questions posed for authentication purpose.

The above discussion indicates a degree of concern from users by introduction of additional processes. The findings support the argument to reduce the number of profile and challenge questions to minimize distraction and enhance usability.

\section{4) Satisfaction}

Satisfaction is one of the important and highly subjective usability attribute [13]. The simplicity and ease of use is an essential and significant criterion for software quality in use identified in ISO standard 9241-11 to assess user satisfaction [2].

Feedback was requested to evaluate student satisfaction on the introduction of multiple choice image questions, overall appearance and use of challenge questions for student authentication in the online examinations. Students' satisfaction in response to statement 13-16 is shown in Table 1 under column satisfaction. Statements on satisfaction factors in 13-15 received a significant degree of agreement and acceptance towards using the image questions. In response to statement 16 on overall satisfaction using challenge questions for security of online learning, there was $29 \%$ disagreement and $13 \%$ neutral response. A total of $58 \%$ agreement is positive, however there is a degree of concern, which could be linked with user distraction reported earlier.

\section{5) Image Questions}

In response to usability, security and privacy concerns reported in earlier studies, we adopted graphical authentication features by using multiple-choice image questions.

The image questions may enhance usability of the PBAF method. Statement on implementation of image questions received $(32+39)$ 71\% agreement. Earlier studies reported graphical authentication to be more usable and secure over text based features [11]. Statement on users' preference of image over text based questions received positive feedback. However, $(3+13) 16 \%$ disagreed and $32 \%$ remained neutral to suggest the use of a hybrid approach to keep the balance.

\section{B. Privacy Results}

Participants' feedback was requested regarding privacy of 28 profile and challenge questions implemented in first phase of user study using the PBAF method in "PHP and MYSQL" online course. The privacy results presented in Table 2 are extracted from participants' feedback to an online questionnaire. Column 1 shows the challenge questions categorized into academic, personal, and favourite themes. Columns 2-4 shows the percentage on 1-3 scales defined earlier and columns 5-7 shows central tendency and dispersion under Mean (M), Median (MD) and Standard Deviation (SD).

\section{1) Academic Questions}

Challenge questions in the academic theme were related to user's previous and current academic information. The academic information is perceived personal and in response to challenge questions "student number", 19\% participants' expressed a great privacy concern and $29 \%$ slight privacy concern. Generic information like student grades was perceived safe with $81 \%$ indicating no privacy concern.

\section{2) Personal Questions}

A large number of questions in the personal theme were reported with some degree of privacy concern. Challenge question "Date of birth" was reported by $(10+45) 55 \%$ participants with a privacy concern including $10 \%$ with a greater degree of privacy concern. Individuals tend to be reluctant to share their personal information online and may require security assurances. Of the three themes, challenge questions in personal theme were reported with the highest privacy concerns compared to academic and favourite themes. 
Table 2 Privacy Survey Results

\begin{tabular}{|c|c|c|c|c|c|c|}
\hline \multirow[t]{2}{*}{ Challenge Questions } & \multicolumn{3}{|c|}{$\begin{array}{c}\% \text { of }[1=\text { Great, } 2=\text { Slight, } 3=\mathrm{No}] \\
\text { Privacy concern }\end{array}$} & \multicolumn{3}{|c|}{$\begin{array}{l}\text { Central Tendency and } \\
\text { Dispersion }(n=31)\end{array}$} \\
\hline & 1 & 2 & 3 & $\mathbf{M}$ & MD & SD \\
\hline \multicolumn{7}{|l|}{ Academic Questions } \\
\hline Student number & 19 & 29 & 52 & 2.3 & 3 & 0.8 \\
\hline Last school attended & 3 & 29 & 68 & 2.6 & 3 & 0.6 \\
\hline Year of graduation from High School & 3 & 29 & 68 & 2.6 & 3 & 0.6 \\
\hline First school attended & 3 & 26 & 71 & 2.7 & 3 & 0.5 \\
\hline Level achieved best grades ever & 6 & 13 & 81 & 2.7 & 3 & 0.6 \\
\hline Grades in highest qualification & 6 & 13 & 81 & 2.7 & 3 & 0.6 \\
\hline First school you attended & 3 & 26 & 71 & 2.7 & 3 & 0.5 \\
\hline \multicolumn{7}{|l|}{ Personal Questions } \\
\hline Date of birth & 10 & 45 & 45 & 2.4 & 2 & 0.7 \\
\hline Best friend's surname & 13 & 19 & 68 & 2.5 & 3 & 0.7 \\
\hline Three letters of your grandfather's surname & 10 & 26 & 64 & 2.5 & 3 & 0.7 \\
\hline Best childhood friend & 16 & 19 & 65 & 2.5 & 3 & 0.8 \\
\hline Three letters of your favourite cousin name & 19 & 13 & 68 & 2.5 & 3 & 0.8 \\
\hline Dream job as a child & 13 & 10 & 77 & 2.6 & 3 & 0.7 \\
\hline Country of your ultimate dream vacation & 13 & 6 & 81 & 2.7 & 3 & 0.7 \\
\hline \multicolumn{7}{|l|}{ Favourite Questions } \\
\hline Childhood place to visit & 16 & 3 & 81 & 2.6 & 3 & 0.8 \\
\hline Book & 10 & 16 & 74 & 2.6 & 3 & 0.7 \\
\hline Childhood hero & 10 & 13 & 77 & 2.7 & 3 & 0.7 \\
\hline Car & 10 & 10 & 80 & 2.7 & 3 & 0.6 \\
\hline Tutor & 19 & 13 & 68 & 2.5 & 3 & 0.8 \\
\hline Animal & 10 & 10 & 80 & 2.7 & 3 & 0.6 \\
\hline Movie & 7 & 16 & 77 & 2.7 & 3 & 0.6 \\
\hline Colour & 13 & 6 & 81 & 2.7 & 3 & 0.7 \\
\hline TV program & 10 & 6 & 84 & 2.7 & 3 & 0.6 \\
\hline Website URL & 10 & 13 & 77 & 2.7 & 3 & 0.7 \\
\hline Food & 3 & 19 & 78 & 2.7 & 3 & 0.5 \\
\hline Car colour & 3 & 13 & 84 & 2.8 & 3 & 0.5 \\
\hline Holiday destination & 6 & 10 & 84 & 2.8 & 3 & 0.6 \\
\hline Academic course & 3 & 16 & 81 & 2.8 & 3 & 0.5 \\
\hline
\end{tabular}

\section{3) Favourite Questions}

Favourite questions were based on individuals' favourites. Based on subjective information, favourite questions may create memorability issues due to more than one possible answers to particular favourites, however, it may reduce the privacy concerns. Out of total 14 favourite questions 12 were reported with no privacy concern by $77 \%$ or more participants. Challenge question favourite "Tutor" was reported with privacy concern by $(19+13) 32 \%$ including $19 \%$ greater privacy concern. Favourite tutor can be perceived as a personal question, hence privacy concern.

\section{CONCLUSION}

Online learning and examination are reported with a number of security, usability and privacy challenges. Student identification is largely reliant upon weak and remote authentication features. The study presented here was part of a multiple phased research work, which implemented a PBAF approach into an online course. Participants feedback based on their experience of using the PBAF method was analyzed and presented.

The quantitative analysis reported here indicate that the PBAF method was effective with potential to improve the appearance and layout. The PBAF method collects answers to text-based personal and image questions for authentication purposes during examination. The number of profile and challenge questions posed during profile building and authentication may be reduced to minimize user distraction. Introduction of image questions enhances the effectiveness with a potential for more research. Overall user satisfaction was positive with more room for improvment to minimize distraction and improve on other usability factors linked with individual challenge questions which are not reported in this paper. 
Particpants expressed some degree of privacy concern in sharing personal and academic information, however, there was little or no privacy concern on sharing questions in the favorite theme. Future work will focus on using activity based questions to mitigate any usability challenges and address privacy issues.

\section{REFERENCES}

[1] Agulla E. G., Rifón L. A., Castro J. L. A., Mateo C. G., editors. "Is My Student at the Other Side? Applying Biometric Web Authentication to ELearning Environments". Eighth IEEE International Conference on Advanced Learning Technologies; 2008: IEEE.

[2] Bevan N. "Usability is quality of use." Advances in Human Factors/Ergonomics. 1995;20:349-54.

[3] Harmon O. R., Lambrinos J., Buffolino J. "Assessment design and cheating risk in online instruction." Online Journal of Distance Learning Administration. 2010;13(3).

[4] Just M., Aspinall D., editors. "Challenging challenge questions". Socio-Economic Strand; 2009: Oxford University UK.

[5] Just M., Aspinall D., editors. "Choosing Better Challenge Questions". Symposium on Usable Privacy and Security (SOUPS); 2009; CA, USA: ACM.

[6] Karaman S. "Examining the effects of flexible online exams on students' engagement in e-learning." Educational Research and Reviews. 2011;6(3):25964.

[7] Nielsen J. "How to conduct a heuristic evaluation." On the World Wide Web. 1994.

[8] Nielsen J., Molich R., editors. "Heuristic evaluation of user interfaces". Proceedings of the SIGCHI conference on Human factors in computing systems: Empowering people; 1990: ACM.

[9] Nielsen J., Tahir M. Homepage usability: 50 websites deconstructed: New Riders Indianapolis, IN; 2002.

[10] Rabkin A., editor. "Personal knowledge questions for fallback authentication: Security questions in the era of Facebook". In SOUPS 2008: Proceedings of the 4th
Symposium on Usable Privacy and Security; 2008; 23, New York, NY, USA: ACM.

[11] Renaud K., Just M., editors. "Pictures or questions?: examining user responses to association-based authentication". Proceedings of the 24th BCS Interaction Specialist Group Conference; 2010: British Computer Society.

[12] Schechter S., Brush A. J. B., Egelman S., editors. "It's No Secret. Measuring the Security and Reliability of Authentication via 'secret' questions". 30th IEEE Symposium on Security and Privacy; 2009: IEEE.

[13] Shneiderman B., Ben S. Designing the user interface: Pearson Education India; 1998.

[14] Standardization I. O. F. Ergonomic Requirements for Office Work with Visual Dispaly Terminals, Part 11: Guidance on Usability. ISO 9241-11. Geneva1998.

[15] Udo G. J. "Privacy and security concerns as major barriers for e-commerce: a survey study." Information Management \& Computer Security. 2001;9(4):16574.

[16] Ullah A., Xiao H., Barker T., Lilley M. "Evaluating security and usability of profile based challenge questions authentication in online examinations." Journal of Internet Services and Applications. 2014;5(1):2.

[17] Ullah A., Xiao H., Lilley M., editors. "Profile Based Student Authentication in Online Examination". International Conference on Information Society 2012; London, UK: IEEE.

[18] Ullah A., Xiao H., Lilley M., Barker T., editors. "Design, privacy and authentication of challenge questions in online examinations". IEEE Conference on e-Learning, e-Managementand and e-Services (IC3e); 2013; Malaysia: IEEE.

[19] Ullah A., Xiao H., Lilley M., Barker T. "Usability of Profile Based Student Authentication and Traffic Light System in Online Examination." The 7th International Conference for Internet Technology and Secured Transactions (ICITST); London, UK: IEEE; 2012. 